\title{
COMPREENSÃO DOS ADOLESCENTES SOBRE A PREVENÇÃO E TRANSMISSÃO DAS INFECÇÕES SEXUALMENTE TRANSMISSÍVEIS EM ESCOLAS DO MUNICÍPIO DE PRESIDENTE MÉDICI, RONDÔNIA, BRASIL
}

\author{
Igor David da COSTA ${ }^{1 *}$ \& Natalia Neto dos Santos NUNES ${ }^{2}$
}

\begin{abstract}
1 Fundação Universidade Federal de Rondônia, Campus Presidente Médici, Departamento de Engenharia de Pesca, Laboratório de Ciências Ambientais. Presidente Médici, Rondônia, Brasil.

2 Fundação Universidade Federal de Rondônia, Campus Rolim de Moura, Programa de Pós-Graduação em Ciência Ambientais. Rolim de Moura, Rondônia, Brasil.

*Autor correspondente: igorbiologia@yahoo.com.br
\end{abstract}

DOI: http://dx.doi.org/10.18571/acbm.118

\section{RESUMO}

A adolescência é a faixa de idade que apresenta a maior incidência de doenças sexualmente transmissíveis, por conta da vivência da sexualidade mais evidenciada nesse período. $\mathrm{O}$ objetivo da pesquisa foi analisar o conhecimento dos adolescentes sobre prevenção, transmissão, manuseio de preservativos e métodos de tratamento das DSTs/AIDS, antes e após oficinas de prevenção. Participaram 501 estudantes da $8^{\mathrm{a}}$ e $9^{\mathrm{a}}$ série do ensino fundamental e ensino médio, da área urbana e rural, de sete escolas do município e distritos de Presidente Médici, Rondônia. Para identificar a diferença do conhecimento dos adolescentes foi utilizado um questionário (pré e pós-teste). Na análise dos dados foi realizado um teste-t de amostras independentes a fim de comparar as variáveis sociodemográficas dos alunos de escolas da zona urbana e rural do município e uma ANOVA twoway para cada variável resposta, com valor de significância de $p \leq 0,05$. A faixa etária concentrouse entre 16 e 18 anos. A maioria os alunos da zona urbana receberam informações sobre o assunto por intermédio da TV e por conversas com seus pais, já para os alunos da zona rural esta foi recebida principalmente pela atuação da escola. A maioria dos entrevistados declarou comprar preservativos, sendo que menos de $23 \%$ solicitam preservativos em postos de saúde do município. Concluímos que há necessidade de um trabalho sistemático, a médio e longo prazo, sobre sexualidade nas escolas para os adolescentes, principalmente para os discentes que frequentam instituições de ensino distantes dos centros urbanos.

Palavras-chave: Doenças sexualmente transmissíveis; AIDS; Área urbana e rural; Amazônia.

\begin{abstract}
Adolescence is the age range that presents the highest incidence of sexually transmitted diseases, due to the experience of sexuality more evidenced in this period. The objective of the study was to analyze adolescents' knowledge about prevention, transmission, condom management and STDs / AIDS treatment methods before and after prevention workshops. 5018 th and 9th grade students from elementary and high school, urban and rural, from seven municipal schools and Presidente Médici districts, Rondônia, participated. To identify the difference in the knowledge of adolescents, a questionnaire was used (pre- and post-test). In the data analysis, a t-test of independent samples was performed in order to compare the socio-demographic variables of students from schools in the urban and rural areas of the municipality and a ANOVA two-way for each response variable, with a significance level of $p \leq 0.05$. The age group was concentrated between 16 and 18 years. Most students in the urban area received information about the subject through TV and conversations with their parents, and for the students in the rural area this was mainly received by the school. Most respondents reported buying condoms, with less than $23 \%$ applying for condoms at municipal health posts. We conclude that there is a need for systematic,
\end{abstract}


medium and long term work on sexuality in schools for adolescents, especially for students who attend educational institutions far from urban centers.

Keywords: Sexually transmitted diseases; AIDS; Urban and rural area; Amazonia.

\section{Introdução}

A adolescência é a faixa de idade que apresenta a maior incidência de doenças sexualmente transmissíveis (DSTs). Aproximadamente, 25\% de todas as DST são diagnosticados em jovens com menos de 25 anos (BRAVERMAN, 2000). A adolescência é um período de transição entre a infância e a idade adulta, caracterizada por intenso crescimento e desenvolvimento, que se manifesta por marcantes transformações anatômicas, fisiológicas, psicológicas e sociais. Essas intensas transformações físicas e biológicas, nesta fase de desenvolvimento humano, influenciam todo o processo psicossocial da formação da identidade do adolescente (BRÊTAS, 2004).

Segundo Brasil (2012), no Brasil entre 2000 e 2011, foi declarado no Sistema de Informação de Mortalidade (SIM) 867 óbitos por hepatites virais, sendo 640 como causa básica e 227 como causa associada, a maioria das quais nas Regiões Nordeste $(31,3 \%)$ e Sudeste $(29,3 \%)$. As DSTs são doenças infecciosas que podem ser disseminadas através do contato sexual. Algumas podem também ser transmitidas por vias não sexuais, que são menos frequentes (OLIVEIRA, 2009). Brasil (2013) afirma que a população sexualmente ativa do Brasil apresenta 937.000 casos de sífilis, 1,5 milhão casos de gonorreia, 1.967.200 casos de clamídia, 640.900 casos de herpes genital e 685.400 casos de HPV. Já o número de HIV positivos registrados pelo Ministério da Saúde chegou a 656.701, desde a década de 1980 até 2012.

Segundo Huh e Cavalini (2013), a maior vulnerabilidade as DSTs e ao vírus do HIV, ocorre devido à liberação sexual, a facilidade dos contatos íntimos e aos estímulos vindos dos meios de comunicação que propiciam os contatos sexuais precoces. A desinformação sobre o assunto devido à diminuição das campanhas educativas, automedicação ou medicação indicada por pessoas não qualificadas, multiplicidade de parceiros, maior liberdade para a prática da atividade sexual em decorrência do uso de métodos anticoncepcionais, dificuldade na investigação dos parceiros sexuais, menor temor do público por essas doenças pela facilidade do diagnóstico e tratamento, facilidade de deslocamento das populações, e por fim o aparecimento da resistência microbiana aos antibióticos e quimioterápicos são alguns fatores que têm contribuído para o aumento da incidência das DSTs (FAÇANHA et al., 2004).

Alguns trabalhos destacaram que os adolescentes possuem maior conhecimento sobre prevenção de DSTs que os adultos, embora essa compreensão seja escassa e insuficiente para promover um comportamento sexual seguro. Entre adolescentes com níveis distintos de conhecimento sobre transmissão e prevenção de DSTs, os que apresentaram maior nível de conhecimento não necessariamente se protegeram do risco de contrair uma infecção (SHRIER, 2004). Embora os adolescentes tenham maior conhecimento sobre DSTs que os adultos, o grau de conhecimento é considerado baixo. Alguns estudos constataram que uma grande proporção de adolescentes se engajam em contatos sexuais, como sexo oral e anal, sem reconhecê-los como fonte de contágio de doenças sexualmente transmissíveis (BOEKELOO e HOWARD, 2002).

Os preservativos masculinos e femininos são a única barreira comprovadamente efetiva contra o HIV e o uso correto e consistente deste método pode reduzir substancialmente o risco de transmissão do HIV e das outras DSTs. O uso regular de preservativos pode levar ao aperfeiçoamento na sua técnica de utilização, reduzindo a frequência de ruptura e escape e, consequentemente, aumentam sua eficácia. Estudos recentes demonstraram que o uso correto e sistemático do preservativo masculino reduz o risco de aquisição do HIV e outras DSTs em até 95\% (FAÇANHA et al., 2004). 
Uma das principais formas de conter o avanço da AIDS e de outras DST é a conscientização da população (CARVALHO et al., 2015). Este trabalho educacional costuma ser feito nas oficinas sobre a prática do sexo seguro, que são de uma forma geral, direcionadas a adolescentes e universitários. As principais estratégias de prevenção, empregadas pelos programas de controle, envolvem a promoção do uso de preservativos, a promoção do uso de agulhas e seringas esterilizadas ou descartáveis, o controle do sangue e derivados, a adoção de cuidados na exposição ocupacional a material biológico e o manejo adequado das outras DSTs (CARVALHO et al., 2015).

A importância de conhecer a população sobre tais tópicos, a fim de promover uma campanha informativa e preventiva acerca das DSTs/AIDS de maneira eficaz e competente, é imprescindível, pois a AIDS não tem cura e a melhor terapêutica ainda é a prevenção. Objetivamos com este estudo avaliar o grau de conhecimento, pré e pós-palestras de adolescentes de escolas públicas de ensino médio e fundamental do município de Presidente Médici, Estado de Rondônia sobre prevenção, transmissão, manuseio de preservativos e métodos de tratamento das DST/AIDS, visando colaborar no desenvolvimento de ações educativas do Projeto de Extensão Universitária Falando de DST/AIDS nas Escolas (PIBEX 2011).

\section{Material e Métodos}

O instrumento de coleta de dados utilizado na pesquisa foi um questionário estruturado, auto-administrado, de múltipla escolha, discursivo e anônimo, composto por perguntas sobre características sócio-demográficas e reprodutivas, conhecimento sobre transmissão e prevenção de DST/AIDS, uso de métodos anticoncepcionais e de preservativo masculino e feminino, além de questões relacionadas a religião, saúde e educação que abordavam aspectos de transmissão e prevenção de AIDS e outras DSTs.

Os questionários foram aplicados em 501 estudantes da $8^{\mathrm{a}}$ e $9^{\mathrm{a}}$ série do ensino fundamental e ensino médio de sete escolas do município e distritos de Presidente Médici, Rondônia (Tabela 1). As escolas selecionadas atendem no turno diurno e noturno. Os alunos da $6^{\mathrm{a}}$ e $7^{\mathrm{a}}$ série não participaram do presente projeto, por apresentarem pouca idade, sendo a aplicação dos questionários não autorizada. Os mesmos questionários foram aplicados antes e depois da realização de uma palestra, que abordou os métodos de transmissão, prevenção, consequências patológicas das DSTs/AIDS e solução de dúvidas a cerca do assunto. A aplicação dos questionários pré e pós-palestras visou avaliar o nível de conhecimento sobre o dado tema e o quanto foi compreendido por parte dos mesmos. Após a revisão dos questionários, os dados foram inseridos e organizados em um banco de dados do programa Excel. Foi realizado um teste-t de amostras independentes visando comparar as variáveis sócio-demográficas dos alunos de escolas da zona urbana e rural do município. As variáveis resposta dos questionários aplicados antes e depois da palestra foram comparadas através de uma ANOVA two-way. As análises foram realizadas no programa Statistica 7.0, sendo as análises inferenciais consideradas significativas com valores de $p \leq 0,05$. 
Tabela 1: Nome das escolas, localização e número total de alunos entrevistados nas escolas de ensino fundamental e médio de Presidente Médica, Rondônia - Brasil.

\begin{tabular}{|c|c|c|}
\hline Nome da Escola & Localidade & $\begin{array}{l}\mathrm{N}^{0} . \text { de } \\
\text { alunos }\end{array}$ \\
\hline Escola Estadual de Ensino Médio Paulo Freire & Município Presidente Médici (zona urbana) & 225 \\
\hline $\begin{array}{l}\text { Escola Estadual de Ensino Médio Carlos Drumond } \\
\text { de Andrade }\end{array}$ & Município Presidente Médici (zona urbana) & 50 \\
\hline $\begin{array}{c}\text { Escola Estadual de Ensino Médio Presidente Emilio } \\
\text { Garrastazul Médici }\end{array}$ & Município Presidente Médici (zona urbana) & 50 \\
\hline Escola Estadual de Ensino Médio Dona Benta & Distrito Novo Riachuelo (zona rural) & 86 \\
\hline Escola Estadual de Ensino Médio Emburana & Distrito Estrela de Rondônia (zona rural) & 37 \\
\hline $\begin{array}{c}\text { Escola Estadual de Ensino Fundamental Dorothy } \\
\text { Mae Stang }\end{array}$ & Assentamento Chico Mendes (zona rural) & 41 \\
\hline $\begin{array}{l}\text { Escola Municipal de Ensino Fundamental Junqueira } \\
\text { Freire } \\
\text { (Extensão da Escola Paulo Freire) }\end{array}$ & Linha 140 (zona rural) & 12 \\
\hline
\end{tabular}

\section{Resultados e Discussão}

A maioria dos alunos (39\%) apresentou idade de 16 a 18 anos, seguido por alunos com idade de 12 a 15 anos (21\%) na zona urbana, para a zona rural a maioria dos alunos apresentou idade entre 16 e 18 anos. Houve predomínio de adolescentes do sexo masculino, seguido do sexo feminino nas escolas da zona urbana e para zona rural o sexo masculino apresentou-se em menor quantidade (Tabela 2).

Com relação ao estado marital a maioria dos alunos afirmaram ser solteiros, em sua maioria encontrados na zona urbana do município. No quesito "conhecimento sobre DST/AIDS", a maior parte dos alunos, que já tinha ouvido falar sobre o determinado assunto eram da zona urbana, todavia mesmo sendo a maioria, um total de $4 \%$ não apresentava conhecimento sobre o assunto. Em sua maioria os alunos da zona urbana receberam esse tipo de informação por intermédio da TV e por conversas com seus pais, já para os alunos da zona rural esta é recebida principalmente pela atuação da escola (Tabela 2). Corroborando com nossos resultados, Oliveira et al. (2009) para estudos no Rio de janeiro, descrevem que a busca de informações por meio dos jornais e das revistas $(18,7 \%)$ foi bastante frequente entre os jovens, bem como pelo rádio e da televisão $(16,9 \%)$ e principalmente a família $(20,4 \%)$.

Os meios de comunicação tornaram-se uma das principais fontes para o jovem no que diz respeito à saúde e à sexualidade. A mídia apresenta de forma frequente imagens ilusórias do que é a sexualidade e do que é uma relação sexual, o que acaba por influenciar diretamente os jovens sobre as formas de prevenção existente e como fazer uso delas ou, até mesmo, como não usá-las (OLIVEIRA et al., 2009). Deve-se ter um olhar voltado para a importância dos meios de comunicação nas atitudes e nas práticas dos adolescentes, uma vez que um número significativo de jovens busca informações nestas fontes (OLIVEIRA et al., 2009).

As informações oriundas do ambiente familiar é um dado surpreendente, pois demonstra um núcleo participativo junto a esses adolescentes. Todavia, a família tanto pode ser um recurso para o crescimento e o desenvolvimento saudável de seus jovens como também pode ser uma limitação nesse processo (PATRÍCIO, 2000). Esta instância social precisa ser apreendida em sua historicidade e permanente transformação, envolvendo finalidades, estruturas, conformações e significados diversos, bem como compromissos mútuos, interações, desempenho de papéis e transmissão de cultura, hábitos, valores e modos de vida (ASSOCIAÇÃO BRASILEIRA DE ENFERMAGEM, 2001).

Relacionado ao questionamento sobre com qual idade receberam informações sobre DST/AIDS, a faixa etária de 11 a 13 anos foi a mais representativa entre ambas as zonas. Pesquisas realizadas por Martins et al. (2006) com adolescentes, envolvendo o conhecimento sobre as DSTs, 
descreveram que em relação a variável faixa etária houve uma predominância de 49,1\% dos adolescentes com idade de 14 anos e 18,2\% de adolescentes de 17 anos. A pesquisa em questão também afirma que há maior participação entre essa faixa etária. Contudo, o boletim epidemiológico sobre HIV/AIDS do Ministério da Saúde (2016), afirmar que na análise da razão de sexos em jovens de 13 a 19 anos, essa era a única faixa etária em que o número de casos HIV era maior entre as mulheres, embora tivessem elevado conhecimento sobre prevenção.

Quando perguntados sobre a iniciação na vida sexual a maioria dos entrevistados, de ambas as zonas, declararam que não haviam realizado até o momento nenhuma prática sexual, sendo este mesmo padrão de resposta encontrado para as perguntas referentes ao já ter sido portador de DSTs e conhecer alguém que é ou foi portador de alguma DSTs (Tabela 2).

Tabela 2: Distribuição percentual dos adolescentes de acordo com as características sociodemográficas e reprodutivas, segundo o tipo de zona no município de Presidente Médici, Rondônia - Brasil (Continua).

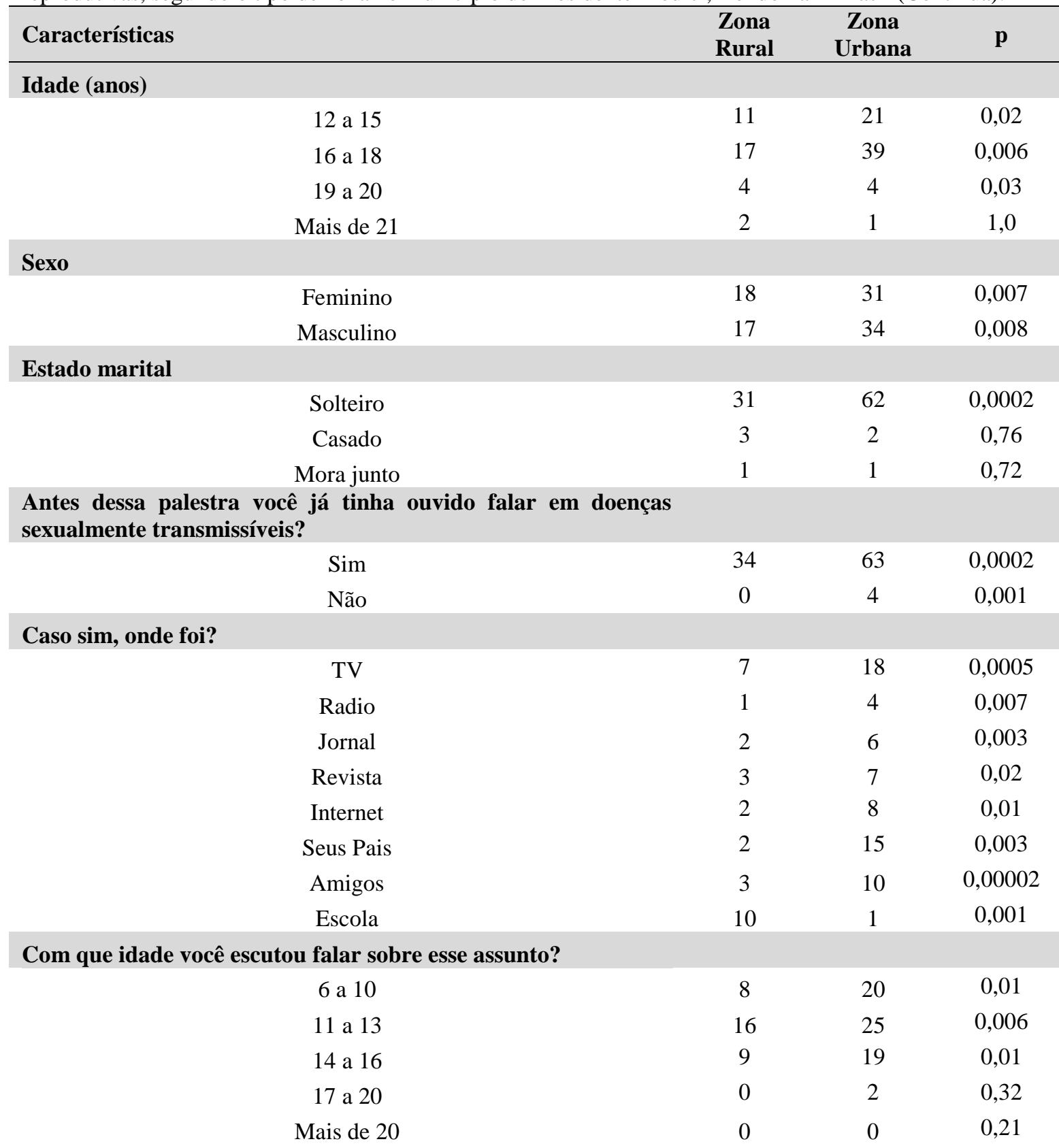


Tabela 2: Distribuição percentual dos adolescentes de acordo com as características sociodemográficas e reprodutivas, segundo o tipo de zona no município de Presidente Médici, Rondônia - Brasil (Conclusão).

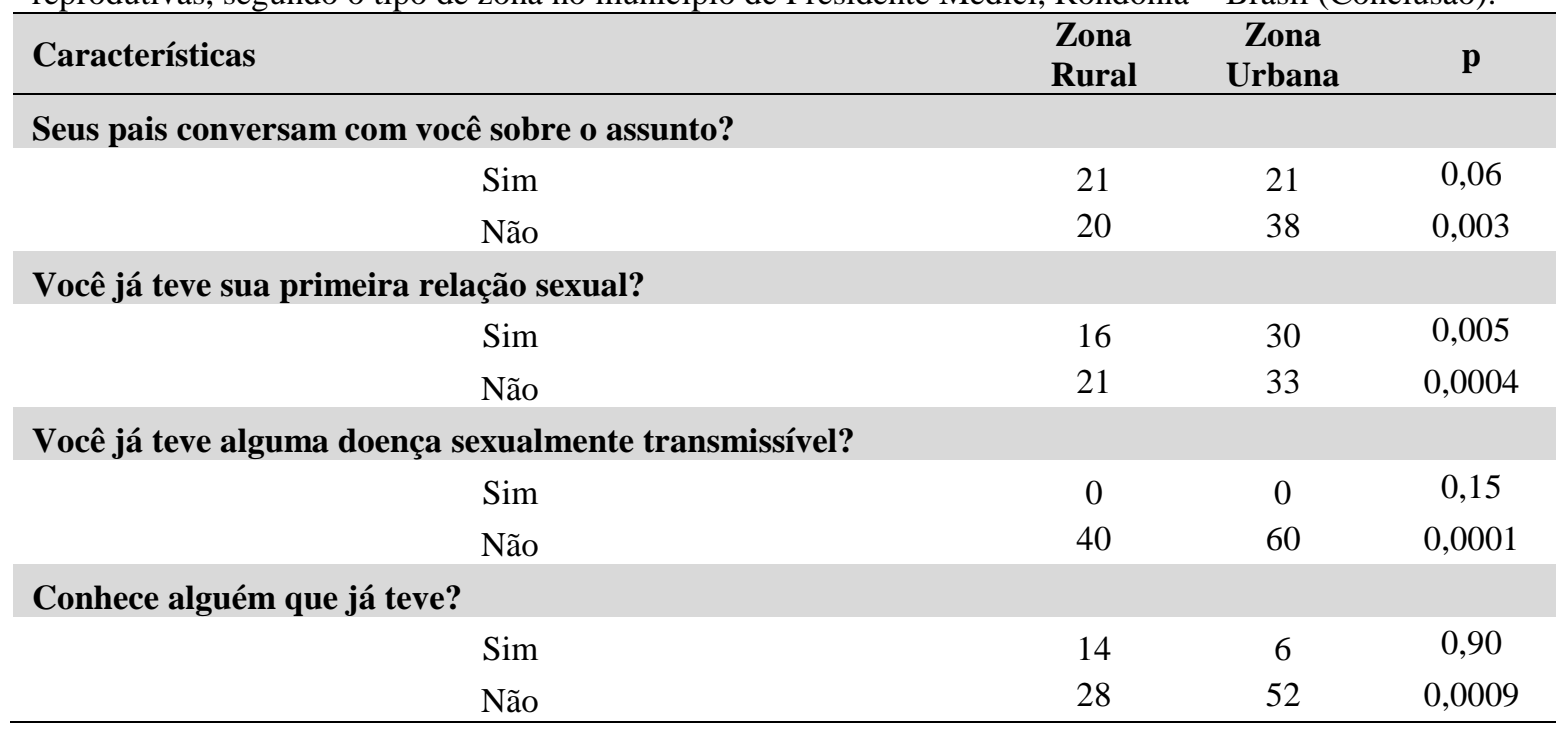

A maioria dos alunos da zona urbana já detinham conhecimento sobre preservativo masculino e feminino antes da realização das palestras, sendo analisado que menos de $42 \%$ dos alunos da zona rural detinham essa informação. Quando perguntados se já tinham realizado algum tipo de relação sexual sem o uso de camisinha, a maioria dos alunos da zona urbana (59\%) e da zona rural (12\%) indicaram fazer uso de preservativo. A maior parte dos estudantes da zona urbana declararam usar camisinha em relações sexuais realizadas atualmente, sendo este percentual seguido pelos alunos da zona rural. Um total de $4 \%$ dos entrevistados da zona rural declarara não usar preservativos nas relações sexuais ocorridas recentemente. Sobre o fato de continuarem usando camisinha em seus relacionamentos, ou seja, uma condição futura referente ao uso de preservativos, o "sim" foi unanime para ambas as zonas. A maioria dos estudantes da zona urbana do município já apresentavam conhecimento sobre DST/AIDS antes das palestras realizadas, sendo menos de $7 \%$ dos alunos de ambas as zonas não conhecedores do tema (Tabela 3 ).

Tabela 3: Distribuição percentual dos adolescentes de acordo com as características sociodemográficas e reprodutivas, segundo o tipo de zona no município de Presidente Médici, Rondônia - Brasil (Continua).

\begin{tabular}{|c|c|c|c|}
\hline $\begin{array}{l}\text { Conhecimento sobre transmissão e prevenção de DST/AIDS } \\
\text { (Continua) }\end{array}$ & $\begin{array}{l}\text { Zona } \\
\text { Rural }\end{array}$ & $\begin{array}{c}\text { Zona } \\
\text { Urbana }\end{array}$ & $\mathbf{p}$ \\
\hline \multicolumn{4}{|l|}{$\begin{array}{l}\text { Antes dessa palestra você já tinha ouvido falar em camisinha } \\
\text { masculina? }\end{array}$} \\
\hline $\operatorname{Sim}$ & 37 & 60 & 0,0003 \\
\hline Não & 0 & 3 & 0,23 \\
\hline \multicolumn{4}{|l|}{$\begin{array}{l}\text { Antes dessa palestra você já tinha ouvido falar em camisinha } \\
\text { feminina? }\end{array}$} \\
\hline Sim & 41 & 49 & 0,01 \\
\hline Não & 1 & 10 & 0,21 \\
\hline \multicolumn{4}{|l|}{ Você já fez sexo sem camisinha? } \\
\hline Sim & 10 & 19 & 0,02 \\
\hline Não & 12 & 59 & 0,0003 \\
\hline \multicolumn{4}{|l|}{ Você atualmente usa camisinha nas suas relações sexuais? } \\
\hline $\operatorname{Sim}$ & 26 & 70 & 0,006 \\
\hline Não & 4 & 0 & 0,005 \\
\hline
\end{tabular}


Tabela 3: Distribuição percentual dos adolescentes de acordo com as características sociodemográficas e reprodutivas, segundo o tipo de zona no município de Presidente Médici, Rondônia - Brasil (Conclusão).

\begin{tabular}{|c|c|c|c|}
\hline Conhecimento sobre transmissão e prevenção de DST/AIDS & $\begin{array}{l}\text { Zona } \\
\text { Rural }\end{array}$ & $\begin{array}{c}\text { Zona } \\
\text { Urbana }\end{array}$ & p \\
\hline \multicolumn{4}{|l|}{$\begin{array}{l}\text { Você pensa em continuar usando camisinha nas suas relações } \\
\text { sexuais? }\end{array}$} \\
\hline Sim & 41 & 59 & 0,0004 \\
\hline Não & 0 & 0 & 0,22 \\
\hline \multicolumn{4}{|l|}{$\begin{array}{l}\text { Antes dessa palestra você sabia quais eram as formas de } \\
\text { transmissão das DSTs/AIDS? }\end{array}$} \\
\hline Sim & 36 & 58 & 0,0003 \\
\hline Não & 3 & 4 & 0,07 \\
\hline
\end{tabular}

De acordo com a tabela 4 , mais de $85 \%$ dos alunos entrevistados apontam que a mãe pode passar o vírus da AIDS para o feto durante o período de gestação. Para a questão relacionado ao bom estado de saúde do individuo frente a contaminação pelo HIV, mais de $89 \%$ dos entrevistados apontaram que a contaminação é positiva. Com relação a contaminação por intermédio da doação de sangue, foi observado um aumento declarado do "não" nos questionários pós-palestra, todavia não foram apresentadas diferenças significativas. Um elevado número de entrevistados (mais de $62 \%$ ) respondeu que a prática de sexo oral sem preservativo transmite o vírus da AIDS. Quando perguntados sobre a utilização de utensílios utilizados em salões de beleza por manicures (alicates de unhas, espátulas e outros), incorretamente esterilizados, uma média de $88 \%$ respondeu que a contaminação era possível. Através da análise dos questionários aplicados, foi observado que um maior número dos entrevistados sabia as formas de tratamento das DST/AIDS.

Segundo os estudos de Cunha et al. (2016) com adolescentes da cidade de Goiânia, estado de Goiás, um total de $94,2 \%$ dos adolescentes entrevistados reconhece a via sexual como forma de transmissão do HIV, afirmando que o uso de preservativos é eficiente na proteção individual contra o vírus e outras DSTs; $96,7 \%$ afirmaram que a via parenteral é uma via importante de transmissão do vírus, enquanto que $92,1 \%$ reconhecem a via vertical como uma das possibilidades de transmissão do vírus. Entretanto, apesar do discernimento da maioria dos adolescentes sobre as vias sexual, parenteral e vertical, 20,3\% afirmaram que o uso de talheres, banheiro, um aperto de mão é uma via eficiente de transmissão do vírus (CUNHA et al., 2016). Nader et al. (2009) verificaram que 8,0\% dos adolescentes entrevistados em uma escola em Canoas, Rio Grande do Sul, ainda acreditam que o beijo e a proximidade de alguém com AIDS consistem em formas de transmissão do vírus. Esses mitos relacionados à transmissão pelo HIV precisam ser desconstruídos no ambiente escolar e destacados pelas autoridades educacionais (MARTINI e BANDEIRA, 2003) uma vez que, quando essas crenças se disseminam facilmente, elas podem fomentar atitudes de cunho preconceituoso (CUNHA et al., 2016).

Quando perguntados se a camisinha somente é utilizada visando a proteção de DST, mais de $77 \%$ dos entrevistados responderam "não", apontando o conhecimento dos mesmos no que tange a utilização de preservativos como método contraceptivo. Mais de $85 \%$ dos entrevistados declaram que não existe cura para AIDS e menos de $15 \%$ apontaram que existe cura para tal doença.

Contrário aos nossos resultados, pesquisas realizadas por Moreira et al. (2012), com alunos de uma escola do interior de Pernambuco, indicaram que muitos alunos declararam que a pílula do dia seguinte é uma forma de se prevenir das DSTs. Em um estudo de avaliação do conhecimento contraceptivo de adolescentes grávidas, Duarte et al. (2012) notaram que mesmo o anticoncepcional oral sendo o mais utilizado, entre aquelas que afirmaram empregar algum método contraceptivo, muitas não tinham ciência do mecanismo de ação do mesmo, com uma parcela representativa das entrevistadas afirmando que o anticoncepcional agia matando espermatozóides. Essa noção pode até mesmo alimentar uma ideia errônea que essa forma de contracepção pode 
auxiliar na eliminação de organismos causadores de DSTs, demonstrando a necessidade de esclarecimentos educativos da função exata desse método (CUNHA et al., 2016). A maior parte da população jovem compreende a importância do uso do preservativo como forma de contracepção e prevenção de DSTs, o que foi observado no presente estudo, diminuindo a probabilidade de contrair essas doenças.

Uma média de $68 \%$ dos jovens entrevistados declarou que a religião é um fator importante na utilização de preservativos, sendo declarado por menos de $35 \%$ dos alunos, que a religião atua como fator negativo na utilização de tal método de proteção. O conceito de religiosidade, ou seja, a adesão à crença e prática relativa a uma igreja ou instituição, pode interferir em comportamentos e respostas individuais. Por serem esses aspectos importantes para a saúde e como sua subjetividade permeia a temática, podemos identificar nesse espaço institucional os vários cenários de Promoção da Saúde (FERREIRA et al. 2012). A religião e a cultura podem ser parceiras na adoção de comportamentos sexuais saudáveis e na superação de situações de vulnerabilidade, mas para tal a enfermagem deve reconhecer seu papel na vida dos jovens, sendo esse reconhecimento fundamental para que educador e educando, juntos, identifiquem estratégias que fortaleçam a autonomia e a conscientização (FERREIRA et al. 2012).

Entre $77 \%$ e $88 \%$ dos entrevistados declararam que compram preservativos, sendo que menos de $23 \%$ solicitam camisinhas em postos de saúde do município. Estudos realizados por Jardim et al. (2013), com adolescentes no município de Campos dos Goytacazes, Rio de Janeiro, apontaram que $9,1 \%$ dos entrevistados alegaram a falta de condições financeiras para adquirir preservativos, evidenciando a carência de informações à cerca das campanhas do Sistema Único de Saúde (SUS) que nos últimos três anos, distribuíram gratuitamente, aproximadamente, 1,3 bilhão de camisinhas por todo o país. "Qualquer pessoa pode retirar o preservativo, sem precisar apresentar qualquer tipo de identificação. Os jovens recebem apoio e informação de profissional para que possam escolher o método contraceptivo mais adequado." (PORTAL DA SAÚDE, 2013).

Quando perguntados sobre a realização de campanhas sobre DST/AIDS e doação de preservativos por parte dos postos de saúde municipais, mais de $94 \%$ dos entrevistados responderam que está seria uma prática positiva na prevenção das DST. Aproximadamente 97\% dos entrevistados julgaram a realização de nossa palestra importante. Não foram encontradas, para nenhuma das perguntas realizadas, diferenças significativas entre os questionários aplicados antes (Pré) e depois (Pós) da palestra sobre DST/AIDS.

Tabela 4: Porcentagem de respostas dos questionários aplicados antes e depois da palestra sobre DST/AIDS e teste de significância de 0,05 ( $\mathrm{n}=501$ ) (Continua).

\begin{tabular}{|c|c|c|c|}
\hline Conhecimento sobre transmissão e prevenção de DST/AIDS & Pré & Pós & $\mathbf{p}$ \\
\hline As mulheres grávidas podem passar o vírus da AIDS para o feto? & & & 0,54 \\
\hline Sim & 89 & 87 & \multirow{3}{*}{0,0001} \\
\hline Não & 11 & 13 & \\
\hline $\mathrm{N}$ & 358 & 410 & \\
\hline \multicolumn{3}{|l|}{ Se você for uma pessoa forte e saudável, você pode pegar AIDS? } & 0,40 \\
\hline Sim & 96 & 89 & \multirow{3}{*}{0,0001} \\
\hline Não & 4 & 11 & \\
\hline $\mathrm{N}$ & 348 & 419 & \\
\hline \multicolumn{3}{|l|}{$\begin{array}{l}\text { Se forem tomados todos os cuidados necessários, uma pode pegar } \\
\text { AIDS ao doar sangue? }\end{array}$} & 0,88 \\
\hline Sim & 50 & 31 & \multirow{3}{*}{0,13} \\
\hline Não & 50 & 69 & \\
\hline $\mathrm{N}$ & 348 & 361 & \\
\hline
\end{tabular}


Tabela 4. Porcentagem de respostas dos questionários aplicados antes e depois da palestra sobre DST/AIDS e teste de significância de 0,05 ( $\mathrm{n}=501$ ) (Conclusão).

\begin{tabular}{|c|c|c|c|}
\hline Conhecimento sobre transmissão e prevenção de DST/AIDS & Pré & Pós & $\mathbf{p}$ \\
\hline Sexo oral transmite AIDS? & & & 0,87 \\
\hline Sim & 68 & 92 & \multirow{3}{*}{0,0002} \\
\hline Não & 32 & 8 & \\
\hline $\mathrm{N}$ & 366 & 382 & \\
\hline $\begin{array}{l}\text { O material usado pela manicure caso não for corretamente } \\
\text { esterilizado pode transmitir AIDS? }\end{array}$ & & & 0,78 \\
\hline Sim & 83 & 94 & \multirow{3}{*}{0,0001} \\
\hline Não & 17 & 6 & \\
\hline $\mathrm{N}$ & 356 & 386 & \\
\hline
\end{tabular}

\begin{tabular}{|c|c|c|c|c|}
\hline \multicolumn{4}{|c|}{$\begin{array}{l}\text { Antes dessa palestra você sabia quais eram as formas de } \\
\text { tratamento das DST/AIDS? }\end{array}$} & 0,82 \\
\hline & Sim & 56 & 76 & \multirow{3}{*}{0,008} \\
\hline & Não & 44 & 24 & \\
\hline & $\mathrm{N}$ & 361 & 379 & \\
\hline \multicolumn{4}{|c|}{ Você acha que a camisinha só evita DST/AIDS? } & 0,64 \\
\hline & $\operatorname{Sim}$ & 15 & 23 & \multirow{3}{*}{0,0001} \\
\hline & Não & 85 & 77 & \\
\hline & $\mathrm{N}$ & 340 & 376 & \\
\hline \multirow[t]{4}{*}{ Existe cura para a AIDS? } & & & & 0,69 \\
\hline & Sim & 15 & 8 & \multirow[t]{3}{*}{0,0001} \\
\hline & Não & 85 & 92 & \\
\hline & $\mathrm{N}$ & 354 & 390 & \\
\hline
\end{tabular}

Você acha que a religião ajuda ou atrapalha no uso da camisinha entre os jovens?

$\begin{array}{cccc}\text { Ajuda } & 71 & 65 & 0,005 \\ \text { Atrapalha } & 29 & 35 & \\ \mathrm{~N} & 350 & 358 & \end{array}$

\section{Você compra ou pede no posto de saúde as camisinhas?}

$350 \quad 358$

$\begin{array}{cccc}\text { Compra } & 88 & 77 & 0,001 \\ \text { Pede } & 12 & 23 & \\ \text { N } & 240 & 273 & \end{array}$

Você acha que os postos de saúde do município deveriam fazer mais campanhas sobre esse assunto?

$\begin{array}{cccc}\text { Sim } & 97 & 98 & 0,001 \\ \text { Não } & 3 & 2 & \\ \text { N } & 343 & 367 & \end{array}$

Você acha que os postos de saúde do município deveriam doar camisinhas?

$\begin{array}{cccc}\text { Sim } & 94 & 96 & 0,0001 \\ \text { Não } & 6 & 4 & \\ \text { N } & 353 & 385 & \end{array}$

\begin{tabular}{ccccc} 
Você acha/achou essa palestra importante? & & & 0,47 \\
Sim & 98 & 97 & 0,0001 \\
Não & 2 & 3 & \\
N & 311 & 388 & \\
\hline
\end{tabular}




\section{Considerações finais}

A realização de palestras junto aos alunos da $8^{\mathrm{a}}$ e $9^{\mathrm{a}}$ série do ensino fundamental e ensino médio das sete escolas do município e distritos de Presidente Médici, Rondônia, permite concluir que o conhecimento desses alunos melhorou em vários aspectos relacionados à sexualidade, gravidez e DSTs. Observamos que os adolescentes se preocupam em aprender mais sobre o seu corpo e o corpo do parceiro.

A atenção primária possibilita a realização da prevenção, que poderá ajudar os adolescentes a desenvolver comportamentos sexuais mais seguros e saudáveis. Dados provenientes desta pesquisa propiciarão o estudo de fatores associados ao maior ou menor conhecimento das DST e aos comportamentos preventivos.

\section{Agradecimentos}

Agradecemos a Pró-reitora de Cultura, Extensão e Assuntos Estudantis - PROCEA/UNIR pela deliberação do Edital PROCEA $n^{\circ}$ 05/2011 e as escolas que colaboraram com o desenvolvimento do projeto.

\section{Referências}

ASSOCIAÇÃO BRASILEIRA DE ENFERMAGEM-ABEN. Projeto Acolher. Adolescer: compreender, atuar, acolher. 1. ed. Brasília: Ministério da Saúde, 2001.

BOEKELOO, B.O.; HOWARD, D.E. Oral sexual experience among young adolescents receiving general health examinations. American Journal Health Behavior, v. 26, p. 306-314, 2002.

BRASIL. Boletim Epidemiológico de Hepatite Virais. 1. ed. Brasília: Ministério da Saúde, 2012.

BRASIL. Departamento de DST, AIDS Hepatites Virais. 1. ed. Brasília: Ministério da Saúde, 2013.

BRAVERMAN, P.K. Sexually transmitted diseases in adolescents. Medical Clinics North America, v. 84, p. 869-889, 2000.

BRÊTAS, J. R. A mudança corporal na adolescência: a grande metamorfose. Temas sobre Desenvolvimento, v. 12. p. 29-38, 2004.

CUNHA, M. P.; DE OLIVEIRA, B. F. R.; DE OLIVEIRA, I. C. M.; PRAXEDES, L. K. S. Análise do conhecimento sobre DSTS/AIDS entre adolescentes em Goiânia, Goiás. Revista da Universidade Vale do Rio Verde, v. 14, p. 650-658, 2016.

CARVALHO, T. G. M. L.; KRABBE, E. C. Ampliando espaços e articulações: o desafio da prevenção as DST, AIDS e hepatites virais no IIEE professor Annes Dias. CATAVENTOSRevista de Extensão da Universidade de Cruz Alta, v. 7, p. 1-16, 2016.

DUARTE, C. F.; HOLANDA, L. B.; MEDEIROS, M. L. Avaliação de conhecimento contraceptivo entre adolescentes grávidas em uma unidade básica de saúde do Distrito Federal. Journal of the Health Sciences Institute, v. 30, p. 140- 143, 2012.

FAÇANHA, M. C.; MENEZES, B. L.; FONTENELE, A. D.; MELO, M. A.; PINHEIRO, A. S.; CARVALHO, C. S.; PEREIRA, L. O. Conhecimento sobre reprodução e sexo seguro de 
adolescentes de uma escola de ensino médio e fundamental de Fortaleza-Ceará. Jornal Brasileiro de Doenças Sexualmente Transmissíveis, v.16, p. 5-9, 2004.

FERREIRA, A. G. N.; SILVA, K. L. D.; SOUSA, P. R. M. D.; GUBERT, F. D. A.; VIEIRA, N. F. C.; PINHEIRO, P. N. D. C. Cultura masculina e religiosidade na prevenção das DST/HIV/AIDS em adolescentes. Revista Mineira de Enfermagem, v.16, p. 578-472, 2012.

HUH, D.M.J; CAVALINI, S.F.S. Recomendações para a Atenção Integral a Adolescentes e Jovens Vivendo com HIV/AIDS / Ministério da Saúde, Secretaria de Vigilância em Saúde, Departamento de DST, AIDS e Hepatites Virais. 1. ed. Brasília: Ministério da Saúde, 2013.

JARDIM, V. M.; JARDIM, L. T. N.; GHETTI, P. A. O.; LAURIANO, M. M.; GADÊLHA, T. A.; SCHMITH, P. M.; MARQUES, A. M. W. D. A. O conhecimento e uso de preservativo por adolescentes: estudo comparativo em uma escola particular e publica. Revista Cientifica da Faculdade de Medicina de Campos, v. 8, 1-8, 2013.

MARTINI, J. G.; BANDEIRA, A. S. Saberes e práticas dos adolescentes na prevenção das Doenças Sexualmente Transmissíveis. Revista Brasileira de Enfermagem, v. 56, p. 160-163, 2003.

MARTINS, L. B. M.; COSTA-PAIVA, L. H. S. D.; OSIS, M. J. D.; SOUSA, M. H. D.; PINTONETO, A. M.; TADINI, V. Fatores associados ao uso de preservativo masculino e ao conhecimento sobre DST/AIDS em adolescentes de escolas públicas e privadas do Município de São Paulo, Brasil. Cadernos de saúde pública, v. 22, p. 315-23, 2006.

MINISTÉRIO

DA

SAÚDE. http://www.aids.gov.br/sites/default/files/anexos/publicacao/2011/50652/boletim_aids_2011_pre liminar3_pdf_20265.pdf. Acessado em 20 de fevereiro de 2016.

MOREIRA, S. B.; PEREIRA, P. S.; BRITO, A. M.; DUARTE, A. E.; BARROS, L. M. DSTs: percepção de estudantes da escola São Vicente de Paula, Exu-PE. Enciclopédia Biosfera, v. 8, p. 2078-2088, 2012.

NADER, S. S.; GERHARDT, C. R.; NADER, P. J. H.; PEREIRA, D. N. Juventude e AIDS: conhecimento entre os adolescentes de uma escola pública em Canoas, RS. Revista da Associação Médica do Rio Grande do Sul, v. 53, p. 374-381, 2009.

OLIVEIRA, D. C. D.; PONTES, A. P. M. D.; GOMES, A. M. T.; RIBEIRO, M. C. M. Conhecimentos e práticas de adolescentes acerca das DST/HIV/AIDS em duas escolas públicas municipais do Rio de Janeiro. Escola Anna Nery Revista de Enfermagem, v. 13, p. 833-41, 2009.

OLIVEIRA, D. C.; PONTES, A. Conhecimentos e Práticas de Adolescentes acerca das DST/HIV/AIDS em duas escolas públicas municipais do Rio de Janeiro. Educação Sexual, v.13, p.53-63, 2009.

PATRÍCIO, Z.M. O cuidado com qualidade de vida dos adolescentes: um movimento ético e estético de "Koans e tricksters". In: Ramos FRS (Organizador), Projeto Acolher: um encontro da enfermagem com adolescente brasileiro. Brasília: ABEn; 2000.p 121-43.

PORTAL DA SAÚDE. http://portalsaude.saude.gov.br/. Acessado em 20 de fevereiro de 2013. 
SHRIER, L A. Sexually transmitted diseases in adolescents: biologic, cognitive, psychologic, behavioral, and social issues. Adolescent Medic Clinic, v. 1, p. 1-22, 2004. 